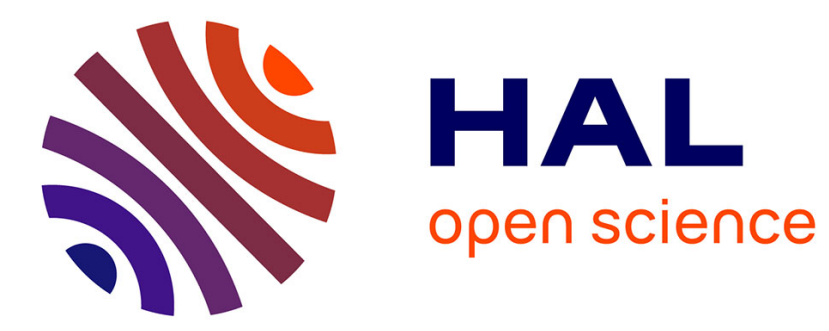

\title{
Electrostatic interactions play a minor role in the binding of ExoS to 14-3-3.
}

Lubna Yasmin, Jeffrey L Veesenmeyer, Maureen H Diaz, Matthew S Francis, Christian Ottmann, Ruth H Palmer, Alan R Hauser, Bengt Hallberg

\section{- To cite this version:}

Lubna Yasmin, Jeffrey L Veesenmeyer, Maureen H Diaz, Matthew S Francis, Christian Ottmann, et al. Electrostatic interactions play a minor role in the binding of ExoS to 14-3-3.. Biochemical Journal, 2010, 427 (2), pp.217-224. 10.1042/BJ20100043 . hal-00479290

\section{HAL Id: hal-00479290 \\ https://hal.science/hal-00479290}

Submitted on 30 Apr 2010

HAL is a multi-disciplinary open access archive for the deposit and dissemination of scientific research documents, whether they are published or not. The documents may come from teaching and research institutions in France or abroad, or from public or private research centers.
L'archive ouverte pluridisciplinaire HAL, est destinée au dépôt et à la diffusion de documents scientifiques de niveau recherche, publiés ou non, émanant des établissements d'enseignement et de recherche français ou étrangers, des laboratoires publics ou privés. 
Electrostatic interactions play a minor role in the binding of ExoS to 14-3-3.

\author{
Lubna Yasmin $^{1^{* \ddagger}}$, Jeffrey L. Veesenmeyer $^{2 *}$, Maureen H Diaz ${ }^{2 \dagger}$ Matthew S. Francis ${ }^{1,3}$ \\ Christian Ottmann ${ }^{4}$, Ruth H Palmer ${ }^{1}$, Alan R Hauser ${ }^{2}$ and Bengt Hallberg ${ }^{1}$ \\ ${ }^{1}$ Department of Molecular Biology, Umeå University, Umeå, Sweden \\ ${ }^{2}$ Department of Microbiology/Immunology, Northwestern University, Chicago, IL, USA \\ ${ }^{3}$ Umeå Centre for Microbial Research, Umeå University, Umeå, Sweden \\ ${ }^{4}$ Chemical Genomics Centre, Otto-Hahn-Strasse 15, Dortmund, Germany
}

Current affiliation: ${ }^{\dagger}$ Centers for Disease Control and Prevention, Atlanta, GA, USA.

${ }^{\ddagger}$ Department of Medical Biosciences/Pathology, Umeå University, Umeå, Sweden

*L.Y. and J.L.V. contributed equally to this study.

Corresponding author:

B. Hallberg,

Department of Molecular Biology,

Building 6L, Umeå University, 90187 Umeå, Sweden

E-mail: bengt.hallberg@molbiol.umu.se

Key Words: ADPRT, ExoS, exoenzyme S, Ras. 14-3-3, PKB/AKT.

Short title: A non-phosphorylated interaction between 14-3-3 and exoS 


\begin{abstract}
:
14-3-3 proteins belong to a family of conserved molecules expressed in all eukaryotic cells that play an important role in a multitude of signaling pathways. 14-3-3 proteins bind to either phosphoserine/phosphothreonine residues or to sequence-specific non-phosphorylated motifs in more than 200 interaction partners (Pozuelo Rubio, Geraghty, Wong, Wood, Campbell, Morrice, Mackintosh. 2004, Biochemical Journal, 379:395-408). These interactions result in cell cycle regulation, apoptosis, stress responses, cell metabolism and malignant transformation. One example of a phosphorylation-independent interaction is the binding of 14-3-3 to exoenzyme S (ExoS), a bacterial ADP-ribosyltransferase toxin of Pseudomonas (P) aeruginosa. Here we have utilized additional biochemical and infection analyses to further define the structural basis of the interaction between ExoS and 14-3-3. An ExoS leucine substitution mutant dramatically reduced the interaction potential with 14-3-3 suggesting that ExoS Leucine residues-422, 423, 426, 428 are important for its interaction with 14-3-3, its enzymatic activity and cytotoxicity. However, ExoS substitution mutants of residues that interact with 14-3-3 through an electrostatic interaction such as Ser-416, His-418, and Asp424 and Asp-427 showed no reduction in their interaction potential with 14-3-3. These ExoS substitution mutants were also as aggressive as wild type ExoS at inducing cell death and to modify endogenous ExoS target within the cell. In conclusion, electrostatic interaction between ExoS and 14-3-3 via polar residues (S416, H418, D424 and D427) appears to be of secondary importance. Thus, the interaction between the "roof" of the groove of 14-3-3 and ExoS relies more on hydrophobic interaction forces, which probably contributes to induce cell death after ExoS infection and activation.
\end{abstract}




\section{Introduction}

The highly conserved eukaryotic protein family 14-3-3 is capable of forming homo- or heterodimers and interacts with a diverse number of target proteins, although it has no enzymatic activity [1-5]. In humans, these partners play a role in physiological processes such as cell proliferation, cell cycle progression, protein trafficking, rearrangement of the cytoskeleton, apoptosis, and survival [6,7]. Much attention has been focused on the conventional interaction between 14-3-3 and specific phosphoserine/phosphothreonine residues located on binding partners. Crystal structure analysis of 14-3-3 in this type of complex with peptides or native partners has revealed a basic amino acid cluster in the amphipathic groove of each monomer that mediates the interaction of 14-3-3 with the phospho-serine/threonine-residues of its binding partners [8, 9]. Each 14-3-3 dimer contains two binding pockets and can interact with canonical binding motifs, defined as mode I (RSXpSXP) and mode II (RXФXpSXP) where $\Phi$ is an aromatic or aliphatic residue and X is any type of residue $[6,9,10]$. From the prominent structural similarity between the $C$ terminal 14-3-3 binding motif of the serotonin-N-acetyltransferase (AANAT, arylalkyl amine$\mathrm{N}$-acetyltranferase) and the plant plasma membrane $\mathrm{H}^{+}$-ATPase, a mode III consensus for 143-3 binding (pS/TX1-2-COOH) has been proposed [11, 12]. Furthermore, additional interactions between 14-3-3 protein and its target partner(s) probably occur outside the amphipathic groove and function to stabilize the three-dimensional arrangement and potentially change the conformation of the target $[8,10,13]$.

However, 14-3-3 also interacts with proteins in a phosphorylation-independent manner, such as human telomerase and p190RhoGEF [14, 15]. Another example is the interaction between 14-3-3 and the specific peptide motif of the bacterial toxin exoenzyme S (ExoS) produced by the opportunistic pathogen $P$ aeruginosa [16-19]. The ExoS toxin is specifically translocated into target eukaryotic cells by the type III secretion system of $P$. aeruginosa and contributes to infections in immuno-compromised individuals including those suffering from cystic fibrosis, cancer, burns, wounds and respiratory compromise requiring mechanical ventilation ([20]and refs. therein). ExoS is regarded as a bifunctional toxin containing N-terminal Rho GTPase activating protein (GAP) activity [21, 22] and C-terminal encoded ADPribosyltransferase activity [23]. The latter activity seems to predominantly and promiscuously modify members of the RAS family [20, 24-26]. Furthermore, the intracellular function of ExoS (specifically the ADP-ribosylation activity) requires an interaction with 14-3-3 and leads to apoptosis by activation of death pathways [17, 19, 27-29]. Further, in a more biological relevant assay the ADP-ribosyltransferase activity of ExoS resulted in increased 
bacterial persistence in the lung and decreased host survival in an animal model of pneumonia [30].

A recent structural analysis of the interaction between 14-3-3 and ExoS showed that the interaction occurs in a reversed orientation relative to previously described 14-3-3/ligand interactions. The binding was described as a non-phospho-mimicking interaction compared with mode I-III and was denoted mode IV. These results suggested that the interaction between ExoS and 14-3-3 was of a hydrophobic nature and that electrostatic forces may not contribute to the overall binding energy between ExoS and 14-3-3 [18]. The hydrophobic residues in the "roof" of the 14-3-3 amphipathic groove (P165, I166, Leu216, I217, L220, and L172) interacted with L422, L423, L426 and L428 of ExoS to provide the main attractive force between the proteins (Figure 1) [18]. This mechanism of binding is different from modes I-III, which predominantly rely on the electrostatic interaction between the phosphoserine/phosphothreonine residues of the client and a basic cluster of amino acids within the amphipathic groove of 14-3-3. In this cluster, two arginines (R56 and R60), one lysine (K49) and one tyrosine (Y128) residue coordinate the phosphate moiety [8, 9]. In ExoS, two negatively charged aspartate residues, D424 and D427, are within the 14-3-3 binding region, but structural analysis indicated that they do not mimic a phosphoryl group, as they are too far away from the positions occupied by the phosphorylated serine or phosphorylated threonine residues during consensus binding in mode I to III motifs [18]. However 14-3-3/ExoS binding was coordinated by several H-bonds, involving S416, H418, D424 and D427 of ExoS and K212, D213, K49 and Y128 of 14-3-3 (Figure 1)[18]. In this study, we have addressed the importance of these ExoS residues for the interaction with 14-33. Employing mutational analysis of ExoS, we performed GST-ExoS pull-down, biochemical, cytotoxicity, and virulence analyses. Our study shows that within the interface of ExoS and 14-3-3, hydrophobic interactions are more important than electrostatic interactions for binding, enzymatic activity and virulence. 


\section{Experimental procedures}

\section{Cell cultures and lysis}

HeLa cells were grown in RPMI 1640 supplemented with $10 \%(\mathrm{v} / \mathrm{v})$ fetal bovine serum and 100 units/mL penicillin. Following bacterial infection, cells were washed in ice-cold $\mathrm{NaCl} / \mathrm{Pi}$ and lysed on ice in lysis buffer [1\%(v/v) Triton x-100, $100 \mathrm{~mm} \mathrm{NaCl}, 50 \mathrm{~mm}$ Tris/HCl (pH 7.5), 1 mm EDTA supplemented with protease inhibitors (Complete, \#1697498, Roche Diagnostics, Basel, Switzerland)]. Lysates were subsequently cleared by centrifugation at $15000 \mathrm{~g}$ for $10 \mathrm{~min}$ at $4{ }^{\circ} \mathrm{C}$. Lysates were pre-cleared with glutathione S-transferase (GST) for $5 \mathrm{~min}$ before incubation with various GST-fusion proteins for $1 \mathrm{~h}$ prior to the addition of Glutathione Sepharose (GE Healthcare, Uppsala, Sweden) for 30 min. After three washes in lysis buffer, samples were boiled for $5 \mathrm{~min}$ in SDS/PAGE sample buffer.

\section{Western analysis, peptides and antibodies}

Anti-14-3-3 (K-19):SC-629 was purchased from Santa Cruz (New York, NY, USA) and monoclonal Ras (cat 610002) was obtained from BD Biosciences (Stockholm, Sweden). RAP, RAS, p-ERK, p-PKB antibodies were obtained from Transduction laboratories (cat: R22020 and R02120) and Cell signaling technologies (cat: 9271 and 9101), respectively. Anti-ExoS was from Agrisera AB, Sweden. Immunoblotting was performed according to the manufacturer's instructions using secondary antibodies conjugated to horseradish peroxidase sheep anti-mouse or rabbit antibodies (Pierce, Rockford, IL, USA, and ECL Plus, AmershamBiosciences).

\section{Plasmids}

pGEX-ExoS(S $\Delta$ ) was constructed as a derivative of pGEX-ExoS(88-453), both of which have been described previously [16]. The substitution mutants (Table 1) pGEXExoS(S416A), pGEX-ExoS(H418A), pGEX-ExoS(S416A;H418A), pGEXExoS(H418A;D424A;D427A) and pGEX-ExoS(L422A;L423A;L426A; L428A) were constructed by digesting pGEX-ExoS (S $\Delta$ ) with NdeI/NheI and PasI/NheI and inserting oligomers (Supplementary Table 2) corresponding to the appropriate amino acid substitutions as outlined in Table 1. All constructs were confirmed by sequencing with DYEnamic ET terminal cycle sequencing kit (Amersham-Biosciences).

\section{Construction of arabinose inducible ExoS derivatives and infection of cells}


Mutant alleles were co-expressed with orf1 to ensure protein stability of full-length ExoS derivatives, encoding the cognate non-secreted chaperone of ExoS. In all cases, DNA was amplified by PCR as described previously [31]. Construction of pMF384 containing arabinose inducible exoS(WT) [16] and pMF516 containing arabinose inducible exoS(LDL426-428) [19] have both been described previously. Arabinose inducible exoS variants on the plasmids pMF743, pMF744, pMF745, pMF746, and pMF747 were obtained by replacing the Cterminal ClaI/KpnI exoS fragment from pMF384 with DNA amplified and restriction enzyme cut with ClaI/KpnI from pGEX-ExoS(S416A), pGEX-ExoS(H418A), pGEX-

ExoS(S416A;H418A), pGEX-ExoS(L422A;L423A;L426A;L428A), and pGEXExoS(H418A;D424A;D427A) respectively (Supplementary Table 2) using the exoS-specific primers, pexoSseq3 (position 973991; forward): 5'-AAGTGATGGCGCTTGGTCT-3' and pexoSd (reverse): 5'-ATGCATGGTACCTCAGGCCAGATCAAGGCCGCG-3'. All constructs were maintained in Escherichia coli DH5 and were confirmed by sequence analysis using the DYEnamic ET terminator cycle sequencing kit (Amersham Biosciences). Stable induction of protein expression in strains grown in the presence of $0.02 \% \mathrm{~L}(+)-$ arabinose was confirmed by western analysis, using polyclonal rabbit anti-ExoS. Bacterial infection of cells was performed in the presence of $0.1 \% \mathrm{~L}(+)$-arabinose, as described previously [26].

\section{Construction and maintenance of $P$. aeruginosa mutants.}

The $P$. aeruginosa strains PA99null, which secretes no known effectors, PA99null $+\mathrm{S}$, which secretes only wild type ExoS, PA99null+S(L426A/D427A/L428A), which secretes ExoS with substitutions in L426, D427, and L428, and PA99null +S(E379A;E381A), which secretes ExoS with substitutions E379 and E381 were generated in previous studies from the clinical strain PA99, which naturally encodes the exoS, exoT and exoU genes but not the exoY gene $[18,30,32]$. Strains secreting the ExoS variants ExoS(L422A), ExoS(L423A), ExoS(L422A/L423A), ExoS(L422A/L423A/L426A/L428A), ExoS(H418A), and ExoS(D424A/D427A) were generated by integrating mutated copies of the exoS allele, under the control of its endogenous promoter, into the chromosome of PA99null. First, specific mutations were generated within the exoS allele of the mini-CTX1-ExoS plasmid [30] using the QuickChange II XL site directed mutagenesis method (Stratagene). Plasmids encoding mutated alleles were then transformed into the Escherichia coli strain S17.1 and subsequently conjugated into the PA99null where the promoter and $\operatorname{exoS}$ sequences were integrated into the Pseudomonas chromosome at the attB locus using the method of Hoang et al. [33]. The 
presence of appropriately mutated sequences was verified by nucleotide sequencing, and protein secretion was verified by growth in low-calcium medium followed by immunoblot screening of culture supernatants using ExoS-specific antibodies. Strains were maintained on Luria-Bertani agar or Vogel-Bonner minimal (VBM) agar. When necessary medium was supplemented with antibiotics at the following concentrations: carbenicillin $500 \mu \mathrm{g} / \mathrm{ml}$, gentamicin $100 \mu \mathrm{g} / \mathrm{ml}$ and tetracycline $100 \mu \mathrm{g} / \mathrm{ml}$.

\section{Mouse model of acute pneumonia}

Studies of acute pneumonia were conducted using the aspiration mouse model described by Comolli et al. [34]. Briefly, bacteria were collected by centrifugation and resuspended to the appropriate concentration in phosphate-buffered saline (PBS). Six- to eight-week-old female $\mathrm{BALB} / \mathrm{c}$ mice were anesthetized by intraperitoneal injection of a mixture of ketamine (100 $\mathrm{mg} / \mathrm{ml})$ and xylazine $(20 \mathrm{mg} / \mathrm{ml})$. Mice were intranasally inoculated with $6 \times 10^{6} \mathrm{CFU}$ of bacteria in $50 \mu \mathrm{L}$ PBS, as determined by optical density. Inocula were confirmed by plating of serial dilutions. Survival was monitored for seven days after infection. Mice were killed when severe illness developed and were scored as dead. Animals were purchased from Harlan and housed in the containment ward of the Center for Comparative Medicine at Northwestern University. All experiments were performed in accordance with the guidelines of the Northwestern University Animal Care and Use Committee. 


\section{Result and discussion}

\section{Leucine residues of ExoS are important for interaction with 14-3-3}

The interaction between 14-3-3 and ExoS is essential for the ADP-ribosyltransferase activity of ExoS [25, 27]. ExoS amino acid residues 419 to 428 are known to be important for this interaction [16]. Although previous pull-down and cytotoxicity analyses indicate that D424 and D427 are not important for the binding of ExoS to 14-3-3 or activation of ExoS-mediated cytotoxicity [19], recent structural analysis indicated that the ExoS peptide is coordinated by H-bonds involving D424 and D427 as well as S416 and H418 of ExoS and D213, K49 and

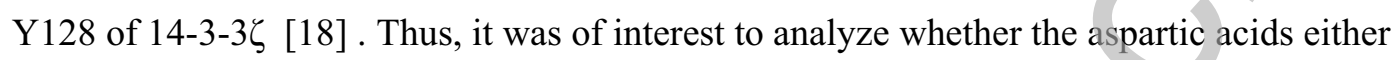
alone or in combination with S416 and H418 were important for the interaction and ExoS enzymatic activity.

A set of single, double, triple, and quadruple substitution ExoS mutants were constructed to investigate which ExoS residues were important for the electrostatic interaction with 14-3-3. Together with previously constructed variants, the new ExoS variants were fused to glutamine S-transferase (GST) giving rise to the following collection of fusion proteins: GST-

ExoS(WT) [17], GST-ExoS(S $\Delta$ ) [16], GST-ExoS(DALDL424-428AAAAA) [19], GSTExoS(L422A;L423A;L426A;L428A), GST-ExoS(D424A; D427A) [19], GSTExoS(H418A;D424A;D427A), GST-ExoS(S416A;H418A), GST-ExoS(S416A), GSTExoS(H418A), GST-ExoS(L422A) [19], GST-ExoS(L423A) [19], GST-ExoS(L426A) [19], and GST-ExoS(L428A) [19] (Table 1). All GST-ExoS derivatives were expressed, purified, and employed in protein pull-down experiments (Figure 2). HeLa cells were harvested and the lysates were pre-cleared with GST beads prior to 1-h incubation with each of the indicated GST-ExoS-fusion proteins. Samples were subsequently washed and run on SDS/PAGE followed by immunoblotting with 14-3-3 antibodies. Since we used a pan-14-3-3 antibody, we did not investigate binding or specificity of different 14-3-3 isoforms in this study. It was observed that GST-ExoS(WT) interacts with 14-3-3, but not GST-beads alone (Figure 2, lane 2 and 3). As previously described, GST-ExoS(DALDL424-428AAAAA) was unable to interact with 14-3-3 proteins from HeLa whole cell lysates (Figure 2, lane 4). Similarly, interaction of GST-ExoS(L422A;L423A;L426A;L428A) with 14-3-3 proteins from HeLa whole cell lysates was clearly reduced (Figure 2, lane 13). The new substitution mutants ExoS(S416A) and ExoS(H418A) agreed with previous data [19] showing that single amino acid substitutions of GST-ExoS between amino acid 419-428 did not obviously reduce their ability to interact with endogenous 14-3-3 proteins (Figure 2, lanes 5 and 6). The double substitution mutants GST-ExoS(D424A; D427A) (Figure 2, lane 12) and GST- 
ExoS(S416A;H418A) (Figure 2, lane 11) and the triple mutant GST-

ExoS(H418A;D424A;D427A) (Figure 2, lane 14) also showed no reduced ability to interact with 14-3-3 proteins. Thus, our pull down analysis strongly suggests that the four leucine residues are important for ExoS mediated interaction with 14-3-3 but that electrostatic interactions play at most a minor role.

Leucine 422, 423, 426, and 428 are important for the induction of cell death by the ADPribosyltransferase domain of ExoS.

A live/dead cell assay was utilized to further investigate the functional significance of the ExoS residues in the 14-3-3 binding domain. HeLa cells were infected for $2 \mathrm{~h}$ with the surrogate bacterium Yersinia pseudotuberculosis lacking endogenous toxins and engineered to express and translocate ExoS (wild type as well as several single, double, triple, and quadruple amino acid substitution variants) into target cells under the control of arabinose $[26,35]$. To ensure that ExoS variants were indeed delivered to infected cells, this assay capitalized on the fact that the GAP activity of ExoS causes transient cell rounding due to disruption of actin microfilaments prior to ADP-ribosylation mediated cell death [36]. Infection with bacteria secreting each of the ExoS variants resulted in a cytotoxic phenotype, (e.g., rounded and semi-detached cells) due to the N-terminal GAP activity of ExoS [21,36], indicating that all ExoS variants were being expressed and injected (data not shown). Both detached and semi-detached cytotoxic cells were washed free from bacteria and transferred to a new Petri dish and incubated overnight with medium containing gentamicin. Cell death was quantified by a trypan blue exclusion assay performed $24 \mathrm{~h}$ after infection. Infection with wild-type ExoS [ExoS(WT)] mediated an irreversible change in cell morphology that ultimately leads to cell death (Figure 3), consistent with the results of previous studies [19, 26]. In fact, when normalized to the survival of uninfected cells, only $9 \%$ of ExoS(WT) infected cells survived (Figure 3). It was also observed that the single substitutions of serine at $416[\operatorname{ExoS}(\mathrm{S} 416 \mathrm{~A})]$ and histidine at 418 [ExoS(H418A)], as well as the double mutant ExoS(S416A;H418A) and the triple mutant ExoS(H418A;D424A;D427A) were as potent as $\operatorname{ExoS}(\mathrm{WT})$ in their ability to induce cell death (Figure 3). These results indicate that negatively charged amino acids at positions 424 and 427 were not crucial for cytotoxicity and, as previously suggested, probably do not mimic phosphorylated serine motifs [18]. The basic charged amino acids at positions 416 and 418, which also were not required for interaction with 14-3-3 proteins, resulted in a phenotype very similar to that of wild type ExoS. However, the quadruple mutant ExoS(L422A;L423A;L426A; L428A), which was unable to 
interact with 14-3-3 proteins in pull-down experiments, was significantly impaired in its ability to induce cell death (93\% survival) (Figure 3 ) after $2 \mathrm{~h}$ infection. Similar results were observed for bacteria expressing the ExoS(LDL426-428AAA) mutant (Figure 3), as previously reported [18]. These results indicate that ExoS(L422A;L423A;L426A;L428A) has reduced ADP-ribosylation activity similar to ExoS(LDL426-428AAA). This phenotype was reminiscent of cells transiently infected with the ADP-ribosylation mutant ExoS(E381A), which recover their original cell structure and morphology overnight due to the transient nature of ExoS GAP-mediated effects [26, 36]. In our initial study of the triple mutant ExoS(LDL426-428AA) [19], the possible involvement of the aspartic acid at position 427 could not be excluded. However, the new quadruple mutant excludes the involvement of aspartic acid 427. Thus, results from the cell death and GST-pull-down assays indicated that the four leucine residues are essential for ExoS activity.

\section{Leu-422, 423, 426, 428 of ExoS are critical for ADP-ribosylation of RAS and RAP1.}

Ras is modified by the ADP-ribosyltransferase activity of ExoS when expressed and delivered into eukaryotic cells by $Y$. pseudotuberculosis devoid of endogenous toxins [26]. This assay was used to further assess the in vivo biological activity of ExoS variants. HeLa cells were infected for $2 \mathrm{~h}$ with $Y$. pseudotuberculosis induced by arabinose to express and translocate ExoS(WT), ExoS(S416A), ExoS(H418A), ExoS(S416A;H418A), ExoS(H418A;D424A;D427A), ExoS(LDL426-428AAA), and ExoS(L422A;L423A;L426A;L428A) into target cells. The cells were then harvested and the resultant lysates were separated by SDS/PAGE followed by immunoblotting with anti-Ras, anti-Rap1 and anti-pan-Erk antibodies as a loading control (Figure 4). As evidenced by an increase in apparent molecular mass, Ras and Rap1 were modified in cells infected with bacteria expressing either wild-type ExoS, ExoS(S416A), ExoS(H418A), ExoS(S416A;H418A) or ExoS(H418A;D424A;D427A) (Figure 4, lanes 3, 5, 6, 7, and 9). Significantly, no modification of Ras and Rap1 were observed in bacteria translocating either ExoS(L422A;L423A;L426A;L428A) or ExoS(LDL426-428AAA) into infected cells (Figure 4 , lanes 4 and 8 ). Thus, the results indicated that loss of the hydrophobic surface of the 14-3-3 binding motif of ExoS dramatically reduces the ability of 14-3-3 to interact with ExoS and act as a cofactor for the ExoS enzymatic activity.

Leu-422, 423, 426, 428 of ExoS are essential for inhibition of Ras activation and Ras downstream signaling. 
Activation of Ras normally results in phosphorylation of the downstream signaling molecules such as Erk and PKB/Akt [26]. Therefore, it was examined if ADP-ribosylation of Ras by ExoS resulted in functional inactivation upon stimulation with EGF of RAS and loss of Erk and $\mathrm{PKB} / \mathrm{Akt}$ phosphorylation (Figure 4). Infection of cells with bacteria expressing wild-type ExoS markedly prevented phosphorylation of Erk and PKB/Akt (Figure 4, lane 3) whereas ExoS(LDL426-428AAA) and ExoS(L422A;L423A;L426A; L428A) caused normal levels of phosphorylation (Figure 4, lanes 4 and 8). Further, all other tested ExoS mutants able to modify Ras and Rap1 [i.e., ExoS(S416A), ExoS(H418A), ExoS(S416A;H418A) and ExoS(H418A;D424A:D427A)] were also able to inhibit phosphorylation of PKB/AKT in a similar manner as ExoS(WT) (Figure 4, lanes 3, 5, 6 ,7, and 9). Thus, S416, H418, D424 and D427 were not critical for interaction between 14-3-3 and ExoS and subsequent ExoS ADPribosyltransferase activity.

\section{Leu-422, 423, 426, 428 are required for ExoS-mediated virulence in a mouse model of acute pneumonia.}

To investigate the biological significance of Leu-422, 423, 426, and 428 of ExoS in a clinically relevant model, we generated $P$. aeruginosa strains that secreted ExoS variants harboring substitutions in residues within the 14-3-3 binding region of ExoS. Variants of the exoS gene were introduced into a neutral site in the chromosome of $P$. aeruginosa strain PA99null, a strain derived from clinical isolate PA99 that does not secrete any type III effectors. ExoS containing a single amino acid substitution (either L422A, L423A, or H418A), two substitutions (L422A/L423A or D424A/D427A), three substitutions (L426A/D427A/L428A) or four substitutions (L422A/L423A/L426A/L428A) were generated and were compared to PA99null (no ExoS), PA99null secreting wild-type ExoS, and PA99null secreting an ExoS variant with two substitutions in the ADP-ribosyltransferase catalytic site (E379A/E381A). To examine the contribution of residues affecting interaction of ExoS with 14-3-3 in pathogenesis of $P$. aeruginosa, mice were intranasally inoculated with $P$. aeruginosa variants and survival was monitored over time. P. aeruginosa secreting ExoS variants L422A, L423A, L422A/L423A, D424A/D427A, or H418A rapidly induced severe illness similar to wild-type ExoS (Figure 5). In contrast, all mice infected with $P$. aeruginosa secreting ExoS(L426A/D427A/L428A) or ExoS(L422A/L423A/L426A/L428A) survived the course of the experiment. The severe attenuation of these strains in mice indicates that the ExoS leucine residues required for interaction with 14-3-3 are relevant in the pathogenesis $P$. aeruginosa infection. These results further support a critical role for ExoS C-terminal leucine 
residues in interaction with 14-3-3 and ExoS activity, contrasting with an at most minimal role for charged residues.

The combination of the above results verifies our hypothesis that hydrophobic interactions are important for both the interaction between 14-3-3 and ExoS and for the ExoS ADPribosyltransferase activity. However, the mechanism by which binding of 14-3-3 activates ExoS remains unclear. It was previously suggested that Leu428 is involved in an ExoS conformational change from an inactive to an active enzyme, but this remains to be investigated [19]. The data clearly suggest that the phosphorylation-independent ExoS/14-3-3 interaction is complex and is likely to involve other interactive surfaces beyond the hydrophobic interaction in the "roof of the groove" of 14-3-3. Further structural and biochemical analyses would enlighten this matter. In conclusion, this study verifies that 14-33 and ExoS interact in a novel fashion that does not involve phospho-mimicking electrostatic interactions but is dependent on the hydrophobic interaction surface between 14-3-3 and ExoS (mode IV). 


\section{Acknowledgements}

Financial support for this work was from the Swedish research council (BH;K2010-68X20427-04-3), and NIH (A.R. H.; R01 AI053674 and K02 AI065615) BH is supported by a grant (08-0597) from the Swedish Cancer Foundation, Lions Cancer Research Foundation, Umeå, and grant (08/084) from the Swedish Childhood Cancer Foundation. RHP is a Swedish Cancer Foundation Research Fellow and is supported by grants from the Swedish Research Council (621-2003-3399), the Swedish Childhood Cancer Foundation (08/074) and the Association for International Cancer Research (AICR 08-0177). 


\section{Figure legends}

Figure 1. Crystal structure of ExoS binding to 14-3-3ఢ. The 14-3-3 interacting motif of ExoS (residues 416-430) is shown as yellow sticks (carbon atoms) with nitrogen and oxygen coloured in blue and red, respectively. 14-3-3 $\zeta$ is shown as grey ribbon with semitransparent

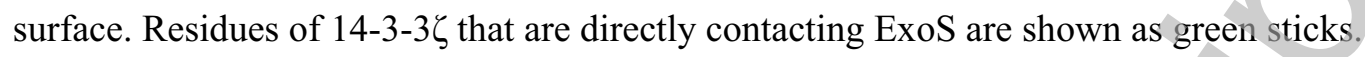
Electrostatic interactions are displayed as dotted lines and the hydrophobic "roof" of the groove of $14-3-3 \zeta$ is shown as green semitransparent surface

Figure 2. Interaction of GST-ExoS variants with endogenous 14-3-3 proteins. HeLa cells were harvested and $1.25 \mu \mathrm{g}$ of lysates were subjected to 'pull-down' analysis with $1.25 \mu \mathrm{g}$ of individual GST-fusion proteins. Samples were separated on a SDS/PAGE followed by immunoblotting with 14-3-3 antibodies. Upper panel: Lane 1, control HeLa cell lysate, $2 \mu \mathrm{g}$; lane 2, GST alone; lane 3, GST-ExoS(WT); lane 4, GST-ExoS(DALDL424-428AAAAA); lane 5, GST-ExoS(S416A); lane 6, GST-ExoS(H418A); lane 7, GST-ExoS(L422A); lane 8, GST-ExoS(L423A); lane 9, GST-ExoS(L426A); lane 10, GST-ExoS(L428A); lane 11, GSTExoS(S416A;H418A); lane 12, GST-ExoS(D424A;D427A); lane 13, GSTExoS(L422A;L423A;L426A;L428A); and lane 14, GST-ExoS(H418A;D424A;D427A). Lower panel: Coomassie blue stained SDS/PAGE showing the purified GST-fusion proteins used in this study. The order corresponds to lanes $2-14$ above.

Figure 3. Cytotoxicity analysis of mammalian cells infected with ExoS variants. Viability of HeLa cells was expressed as survival percentage after $24 \mathrm{~h}$ intoxication with ExoS variants. HeLa cells were infected for $2 \mathrm{~h}$ in the presence of $0.1 \% \mathrm{~L}(+)$-arabinose with Yersinia (YPIII/pIB251) carrying plasmids expressing different ExoS variants under the control of arabinose-inducible promoters as indicated in the figure. Both detached and semi-detached cytotoxic cells were washed free from bacteria and transferred to a new Petri dish and incubated overnight with fresh medium containing gentamicin. A trypan blue exclusion assay was performed $24 \mathrm{~h}$ after infection to quantify the percentage of dead cells. Error bars represent s.e.m. Each bar represents the mean values of three independent experiments.

Figure 4. Protein modification in host mammalian cells following infection with variants of ExoS. HeLa cells were infected for $2 \mathrm{~h}$ in the presence of $0.1 \% \mathrm{~L}(+)$-arabinose with Yersinia (YPIII/pIB251) carrying plasmids expressing different ExoS variants under the control of 
arabinose-inducible promoters. Lane 1, uninfected cells; lane 2, unifected cells, lane 3, YPIII(pMF384) expressing ExoS(WT); lane 4, YPIII(pMF516) expressing ExoS(LDL426428AAA); lane 5, YPIII(pMF743) expressing ExoS(S416A); lane 6, YPIII(pMF744) expressing ExoS(H418A); lane 7, YPIII(pMF745) expressing ExoS(S416A;H418A); lane 8, YPIII(pMF746) expressing ExoS(L422A;L423A;L426A;L428A) and lane 9, YPIII(pMF747) expressing ExoS(H418A;D424A;D427A). ExoS-mediated ADP-ribosylation of HeLa cell proteins following infection and stimulation with EGF $(50 \mathrm{ng} / \mathrm{ml})$ for $3 \mathrm{~min}$ before harvesting, as indicated in figure. HeLa cells were lysed and western blot analysis was performed on immunoblotted filters with anti-RAS, anti-RAP1, anti-phospho-ERK1/2, anti-phospho$\mathrm{PKB} / \mathrm{Akt}$, and anti-pan-ERK antibodies as indicated in figure.

Figure 5: Survival of mice infected with $P$. aeruginosa secreting ExoS variants. Mice were infected with strain PA99null secreting the indicated ExoS variant. Each symbol represents the number of mice surviving in each experimental group over time. Results are from a representative experiment $(n=5)$; similar results were obtained in at least two independent experiments. Survival of mice infected with PA99null + ExoS(L426A/D427A/L428A) or PA99null+ExoS(L422A/L423A/L426A/L428A) differed significantly from that of mice infected with PA99null+ExoS $(P<0.01, \log$ rank test for equality of survivor functions). 


\section{REFERENCES.}

1 Aitken, A. (2006) 14-3-3 proteins: a historic overview. Semin Cancer Biol. 16, $162-$ 172

2 Benzinger, A., Popowicz, G. M., Joy, J. K., Majumdar, S., Holak, T. A. and Hermeking, H. (2005) The crystal structure of the non-liganded 14-3-3sigma protein: insights into determinants of isoform specific ligand binding and dimerization. Cell Res. 15, 219-227 3 Jin, J., Smith, F. D., Stark, C., Wells, C. D., Fawcett, J. P., Kulkarni, S., Metalnikov, P., O'Donnell, P., Taylor, P., Taylor, L., Zougman, A., Woodgett, J. R., Langeberg, L. K., Scott, J. D. and Pawson, T. (2004) Proteomic, functional, and domain-based analysis of in vivo 14-3-3 binding proteins involved in cytoskeletal regulation and cellular organization. Curr Biol. 14, 1436-1450

4 Meek, S. E., Lane, W. S. and Piwnica-Worms, H. (2004) Comprehensive proteomic analysis of interphase and mitotic 14-3-3-binding proteins. J Biol Chem. 279, 32046-32054 5 Pozuelo Rubio, M., Geraghty, K. M., Wong, B. H., Wood, N. T., Campbell, D. G., Morrice, N. and Mackintosh, C. (2004) 14-3-3-affinity purification of over 200 human phosphoproteins reveals new links to regulation of cellular metabolism, proliferation and trafficking. Biochem J. 379, 395-408

6 Mackintosh, C. (2004) Dynamic interactions between 14-3-3 proteins and phosphoproteins regulate diverse cellular processes. Biochem J. 381, 329-342

7 Morrison, D. K. (2009) The 14-3-3 proteins: integrators of diverse signaling cues that impact cell fate and cancer development. Trends Cell Biol. 19, 16-23

8 Rittinger, K., Budman, J., Xu, J., Volinia, S., Cantley, L. C., Smerdon, S. J., Gamblin, S. J. and Yaffe, M. B. (1999) Structural analysis of 14-3-3 phosphopeptide complexes identifies a dual role for the nuclear export signal of 14-3-3 in ligand binding. Mol Cell. 4, 153-166

9 Yaffe, M. B., Rittinger, K., Volinia, S., Caron, P. R., Aitken, A., Leffers, H., Gamblin, S. J., Smerdon, S. J. and Cantley, L. C. (1997) The structural basis for 14-3-3:phosphopeptide binding specificity. Cell. 91, 961-971

10 Coblitz, B., Wu, M., Shikano, S. and Li, M. (2006) C-terminal binding: an expanded repertoire and function of 14-3-3 proteins. FEBS Lett. 580, 1531-1535

11 Ganguly, S., Weller, J. L., Ho, A., Chemineau, P., Malpaux, B. and Klein, D. C. (2005) Melatonin synthesis: 14-3-3-dependent activation and inhibition of arylalkylamine Nacetyltransferase mediated by phosphoserine-205. Proc Natl Acad Sci U S A. 102, 1222-1227 
12 Wurtele, M., Jelich-Ottmann, C., Wittinghofer, A. and Oecking, C. (2003) Structural view of a fungal toxin acting on a 14-3-3 regulatory complex. EMBO J. 22, 987-994

13 Obsil, T., Ghirlando, R., Anderson, D. E., Hickman, A. B. and Dyda, F. (2003) Two 14-3-3 binding motifs are required for stable association of Forkhead transcription factor FOXO4 with 14-3-3 proteins and inhibition of DNA binding. Biochemistry. 42, 15264-15272 14 Seimiya, H., Sawada, H., Muramatsu, Y., Shimizu, M., Ohko, K., Yamane, K. and Tsuruo, T. (2000) Involvement of 14-3-3 proteins in nuclear localization of telomerase.

EMBO J. 19, 2652-2661

15 Zhai, J., Lin, H., Shamim, M., Schlaepfer, W. W. and Canete-Soler, R. (2001)

Identification of a novel interaction of 14-3-3 with p190RhoGEF. J Biol Chem. 276, 4131841324

16 Henriksson, M. L., Francis, M. S., Peden, A., Aili, M., Stefansson, K., Palmer, R., Aitken, A. and Hallberg, B. (2002) A nonphosphorylated 14-3-3 binding motif on exoenzyme $\mathrm{S}$ that is functional in vivo. Eur J Biochem. 269, 4921-4929

17 Henriksson, M. L., Troller, U. and Hallberg, B. (2000) 14-3-3 proteins are required for the inhibition of Ras by exoenzyme S. Biochem J. 349 Pt 3, 697-701

18 Ottmann, C., Yasmin, L., Weyand, M., Veesenmeyer, J. L., Diaz, M. H., Palmer, R. H., Francis, M. S., Hauser, A. R., Wittinghofer, A. and Hallberg, B. (2007) Phosphorylationindependent interaction between 14-3-3 and exoenzyme $S$ : from structure to pathogenesis. EMBO J. 26, 902-913

19 Yasmin, L., Jansson, A. L., Panahandeh, T., Palmer, R. H., Francis, M. S. and Hallberg, B. (2006) Delineation of exoenzyme S residues that mediate the interaction with 143-3 and its biological activity. FEBS J. 273, 638-646

20 Hauser, A. R. (2009) The type III secretion system of Pseudomonas aeruginosa: infection by injection. Nat Rey Microbiol. 7, 654-665

21 Goehring, U. M., Schmidt, G., Pederson, K. J., Aktories, K. and Barbieri, J. T. (1999) The N-terminal domain of Pseudomonas aeruginosa exoenzyme $\mathrm{S}$ is a GTPase-activating protein for Rho GTPases. J Biol Chem. 274, 36369-36372

22 Wurtele, M., Renault, L., Barbieri, J. T., Wittinghofer, A. and Wolf, E. (2001)

Structure of the ExoS GTPase activating domain. FEBS Lett. 491, 26-29

23 Knight, D. A., Finck-Barbancon, V., Kulich, S. M. and Barbieri, J. T. (1995)

Functional domains of Pseudomonas aeruginosa exoenzyme S. Infect Immun. 63, 3182-3186 
24 Coburn, J., Dillon, S. T., Iglewski, B. H. and Gill, D. M. (1989) Exoenzyme S of Pseudomonas aeruginosa ADP-ribosylates the intermediate filament protein vimentin. Infect Immun. 57, 996-998

25 Coburn, J. and Gill, D. M. (1991) ADP-ribosylation of p21 ras and related proteins by Pseudomonas aeruginosa exoenzyme S. Infect Immun. 59, 4259-4262

26 Henriksson, M. L., Sundin, C., Jansson, A. L., Forsberg, A., Palmer, R. H. and Hallberg, B. (2002) Exoenzyme S shows selective ADP-ribosylation and GTPase-activating protein (GAP) activities towards small GTPases in vivo. Biochem J. 367, 617-628

27 Fu, H., Coburn, J. and Collier, R. J. (1993) The eukaryotic host factor that activates exoenzyme $\mathrm{S}$ of Pseudomonas aeruginosa is a member of the 14-3-3 protein family. Proc Natl Acad Sci U S A. 90, 2320-2324

28 Jansson, A. L., Yasmin, L., Warne, P., Downward, J., Palmer, R. H. and Hallberg, B. (2006) Exoenzyme $\mathrm{S}$ of Pseudomonas aeruginosa is not able to induce apoptosis when cells express activated proteins, such as Ras or protein kinase B/Akt. Cell Microbiol. 8, 815-822

29 Kaufman, M. R., Jia, J., Zeng, L., Ha, U., Chow, M. and Jin, S. (2000) Pseudomonas aeruginosa mediated apoptosis requires the ADP-ribosylating activity of exoS. Microbiology. 146 ( Pt 10), 2531-2541

30 Shaver, C. M. and Hauser, A. R. (2004) Relative contributions of Pseudomonas aeruginosa ExoU, ExoS, and ExoT to virulence in the lung. Infect Immun. 72, 6969-6977 31 Francis, M. S. and Wolf-Watz, H. (1998) YopD of Yersinia pseudotuberculosis is translocated into the cytosol of HeLa epithelial cells: evidence of a structural domain necessary for translocation. Mol Microbiol. 29, 799-813

32 Feltman, H., Schulert, G., Khan, S., Jain, M., Peterson, L. and Hauser, A. R. (2001) Prevalence of type III secretion genes in clinical and environmental isolates of Pseudomonas aeruginosa. Microbiology. 147, 2659-2669

33 Hoang, T. T., Kutchma, A. J., Becher, A. and Schweizer, H. P. (2000) Integrationproficient plasmids for Pseudomonas aeruginosa: site-specific integration and use for engineering of reporter and expression strains. Plasmid. 43, 59-72

34 Comolli, J. C., Hauser, A. R., Waite, L., Whitchurch, C. B., Mattick, J. S. and Engel, J. N. (1999) Pseudomonas aeruginosa gene products PilT and PilU are required for cytotoxicity in vitro and virulence in a mouse model of acute pneumonia. Infect Immun. 67, $3625-3630$ 
35 Francis, M. S., Lloyd, S. A. and Wolf-Watz, H. (2001) The type III secretion chaperone LcrH co-operates with YopD to establish a negative, regulatory loop for control of Yop synthesis in Yersinia pseudotuberculosis. Mol Microbiol. 42, 1075-1093

36 Frithz-Lindsten, E., Du, Y., Rosqvist, R. and Forsberg, A. (1997) Intracellular targeting of exoenzyme S of Pseudomonas aeruginosa via type III-dependent translocation induces phagocytosis resistance, cytotoxicity and disruption of actin microfilaments. Mol Microbiol. 25, 1125-1139

37 Henriksson, M. L., Rosqvist, R., Telepnev, M., Wolf-Watz, H. and Hallberg, B. (2000) Ras effector pathway activation by epidermal growth factor is inhibited in vivo by exoenzyme S ADP-ribosylation of Ras. Biochem J. 347 Pt 1, 217-222

38 Rosqvist, R., Forsberg, A., Rimpilainen, M., Bergman, T. and Wolf-Watz, H. (1990)

The cytotoxic protein YopE of Yersinia obstructs the primary host defence. Mol Microbiol. 4, 657-667 
Table 1: Summary of recombinant ExoS expression plasmids and bacterial strains used in this study. Substituted amino acid(s) are underlined. With respect to the GST-ExoS fusions, GST-ExoS(88-453) was the parental allele (WT) such that all other alleles listed differ only by the amino acid substitution indicated in parentheses. The number in front of plasmid indicates the serial number.

\begin{tabular}{|c|c|c|}
\hline Plasmid or Bacterial strain (mutation) & Substituted amino acid(s) & Reference \\
\hline 1. GST alone & Amersham & \\
\hline 2. GST-ExoS(88-453), wild type & $\mathrm{S}^{416}$ GHSQGLLDALDL ${ }^{428}$ & {$[37]$} \\
\hline 3. GST-ExoS(88-453; S $\Delta)$ & $\mathrm{M}^{419} \mathrm{AAAA}^{428}$ & {$[16]$} \\
\hline 4. GST-ExoS(H418A;D424A;D427A) & $\mathrm{S}^{416} \mathrm{G}^{\mathrm{A} S Q G L L} \underline{\underline{A} A L A L^{428}}$ & This study \\
\hline 5. GST-ExoS(L422A;L423A;L426A;L428A) & $\mathrm{S}^{416} \mathrm{GHSQGAADAADA}^{428}$ & This study \\
\hline 6. GST-ExoS(DALDL424-428AAAAA) & $\mathrm{S}^{416}$ GHSQGLLAAAAA ${ }^{428}$ & [19] \\
\hline 7. GST-ExoS(D424A;D427A) & $\mathrm{S}^{416} \mathrm{GHSQGLLAALAL}{ }^{428}$ & [19] \\
\hline 8. GST-ExoS(S416A) & $\underline{A}^{416}$ GHSQGLLDALDL $^{428}$ & This study \\
\hline 9. GST-ExoS(H418A) & $\mathrm{S}^{416} \mathrm{GASQGLLDALDL}^{428}$ & This study \\
\hline 10. GST-ExoS(L422A) & $\mathrm{S}^{416} \mathrm{GHSQGALDALDL}{ }^{428}$ & {$[19]$} \\
\hline 11. GST-ExoS(L423A) & $\mathrm{S}^{416} \mathrm{GHSQGLADALDL}^{428}$ & [19] \\
\hline 12. GST-ExoS(L426A) & $\mathrm{S}^{416}$ GHSQGLLDAADL $^{428}$ & [19] \\
\hline 13. GST-ExoS(L428A) & $\mathrm{S}^{416} \mathrm{GHSQGLLDALDA}^{428}$ & [19] \\
\hline 14. Mini-CTX1-ExoS, wild type & $\mathrm{S}^{416}$ GHSQGLLDALDL ${ }^{428}$ & [28] \\
\hline 15. Mini-CTX1-ExoS (L422A) & $\mathrm{S}^{416} \mathrm{GHSQGALDALDL}^{428}$ & This study \\
\hline 16. Mini-CTX1-ExoS (L423A) & $\mathrm{S}^{416} \mathrm{GHSQGLADALDL}^{428}$ & This study \\
\hline 17. Mini-CTX1-ExoS (L422A;L423A) & $\mathrm{S}^{416}$ GHSQGAADALDL ${ }^{428}$ & This study \\
\hline $\begin{array}{l}\text { 18. Mini-CTX1-ExoS } \\
\text { (L422A;L423A;L426A; } \\
\text { L428A) }\end{array}$ & $\mathrm{S}^{416} \mathrm{GHSQG} \underline{\underline{\mathrm{AA}} \mathrm{DA}} \underline{\mathrm{AD}} \underline{\mathrm{A}}^{428}$ & This study \\
\hline 19. Mini-CTX1-ExoS (H418A) & $\mathrm{S}^{416} \mathrm{GASQGLLDALDL}^{428}$ & This study \\
\hline 20. Mini-CTX1-ExoS (D424A;D427A) & $\mathrm{S}^{416} \mathrm{GHSQGLLAALAL}^{428}$ & This study \\
\hline pMF384 (arabinose inducible exo $S$ (WT) & $\mathrm{S}^{416}$ GHSQGLLDALDL $^{428}$ & {$[16]$} \\
\hline $\begin{array}{l}\text { pMF516 (arabinose inducible exoS (LDL426- } \\
\text { 428AAA) }\end{array}$ & $\mathrm{S}^{416} \mathrm{GHSQGLLDAAAA}^{428}$ & [19] \\
\hline pMF743 (arabinose inducible exoS (S416A) & $\mathrm{A}^{416} \mathrm{GHSQGLLDALDL}^{428}$ & This study \\
\hline pMF744 (arabinose inducible exoS (H418A) & $\overline{\mathrm{S}}^{416} \mathrm{GASQGLLDALDL}^{428}$ & This study \\
\hline $\begin{array}{l}\text { pMF745 (arabinose inducible exoS } \\
\text { (S416A;H418A) }\end{array}$ & $\underline{\mathrm{A}}^{416}$ GASQGLLDALDL ${ }^{428}$ & This study \\
\hline $\begin{array}{l}\text { pMF746 (arabinose inducible exoS } \\
\text { (L422A;L423A;L426A;L428A) }\end{array}$ & $\mathrm{S}^{416} \mathrm{GHSQG} \underline{\underline{A A D A}} \underline{\mathrm{ADD}} \underline{A}^{428}$ & This study \\
\hline $\begin{array}{l}\text { pMF747 (arabinose inducible exoS } \\
\text { (H418A;D424A;D427A) }\end{array}$ & $\mathrm{S}^{416}$ GÁSQGLLAALALLL ${ }^{428}$ & This study \\
\hline \multicolumn{3}{|l|}{ P. aeruginosa strains } \\
\hline 1. PA99 $\triangle$ UTS/ExoS, wild type & $\mathrm{S}^{416} \mathrm{GHSQGLLDALDL}^{428}$ & {$[28]$} \\
\hline 2. PĀ990UTS/ExoS (E379A;E381A) & $\mathrm{S}^{416} \mathrm{GHSQGLLDALDL}^{428}$ & {$[16]$} \\
\hline 3. PA99 $\Delta U T S / E x o S ~(L 426 A ; D 427 A ; L 428 A)$ & $\mathrm{S}^{416}$ GHSQGLLDAAAA $^{428}$ & {$[16]$} \\
\hline 4. PA99AUTS/ExoS (L422A) & $\mathrm{S}^{416} \mathrm{GHSQGALDALDL}^{428}$ & This study \\
\hline 5. PA99AUTS/ExoS (L423A) & $\mathrm{S}^{416}$ GHSQGLADALDL $^{428}$ & This study \\
\hline
\end{tabular}




\begin{tabular}{|c|c|c|}
\hline 6. PA99AUTS/ExoS (L422A;L423A) & $\mathrm{S}^{416}$ GHSQGAADALDL $^{428}$ & This study \\
\hline $\begin{array}{l}\text { 7. PA99AUTS/ExoS (L422A;L423A;L426A; } \\
\text { L428A) }\end{array}$ & $\mathrm{S}^{416} \mathrm{GHSQGAADAAD}^{428}$ & This study \\
\hline 8. PA99AUTS/ExoS (H418A) & $\mathrm{S}^{416} \mathrm{GASQGLLDALDL}^{428}$ & This study \\
\hline 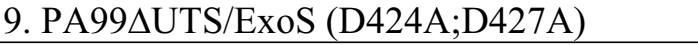 & $\mathrm{S}^{416}$ GHSQGLLAALAL ${ }^{428}$ & This study \\
\hline Y. pseudotuberculosis strain & & \\
\hline YPIII/pIB251 ( & & [38] \\
\hline
\end{tabular}

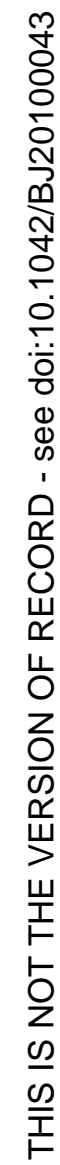




\section{B Biochemical Journal Immediate Publication. Published on 09 Feb 2010 as manuscript BJ20100043}

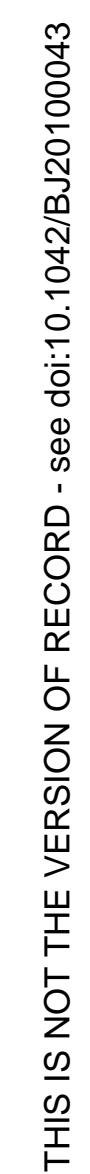

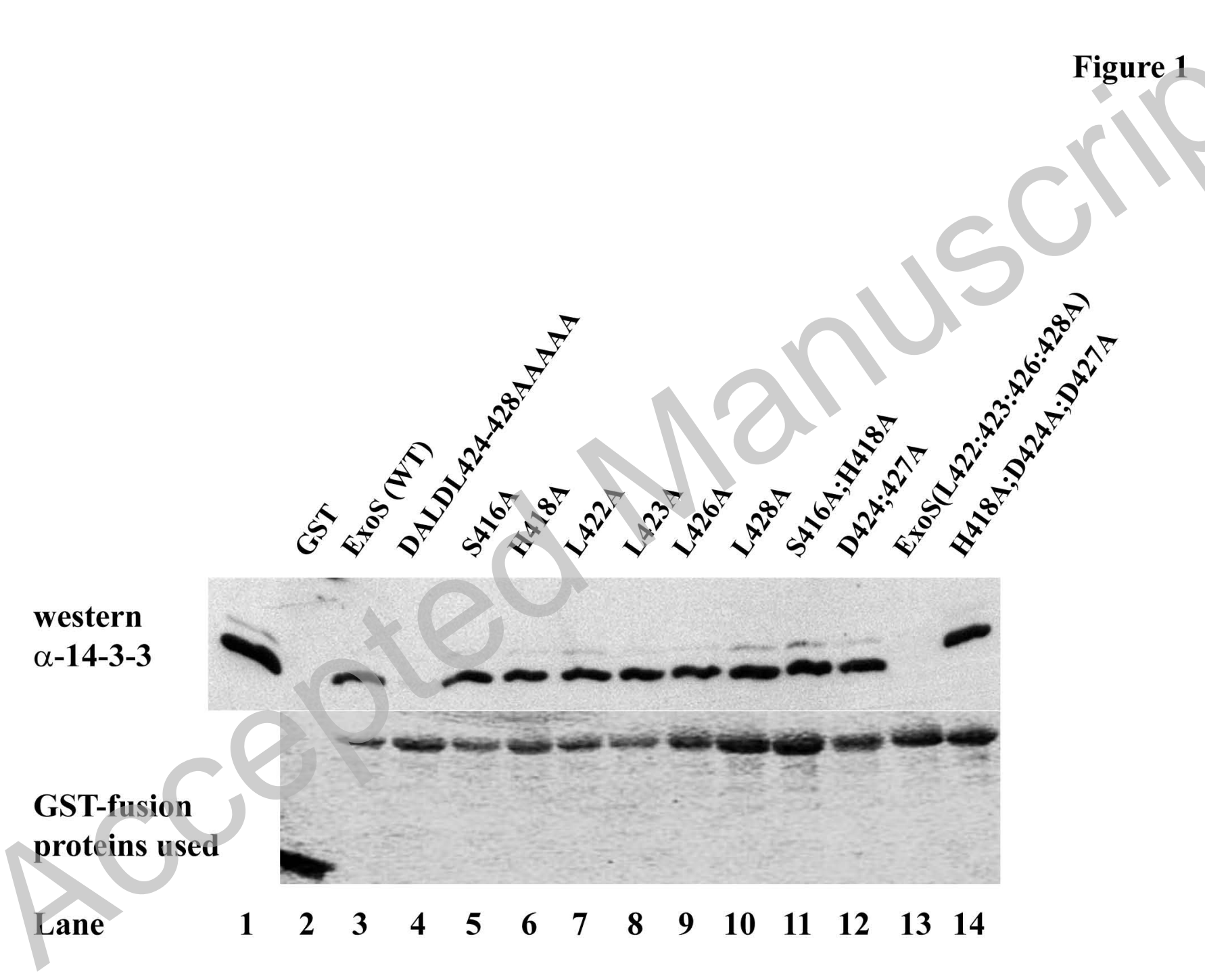


Figure 3

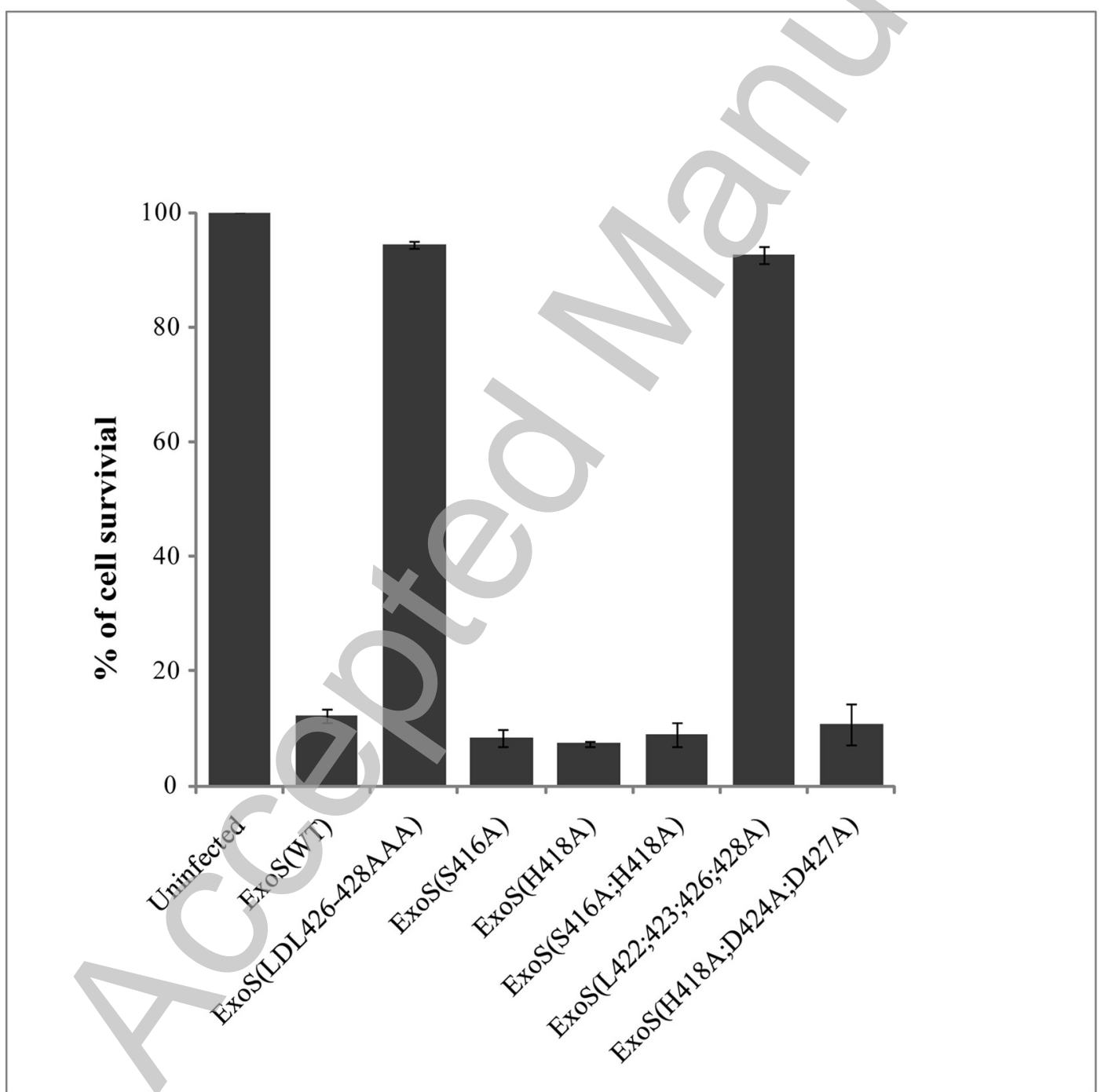




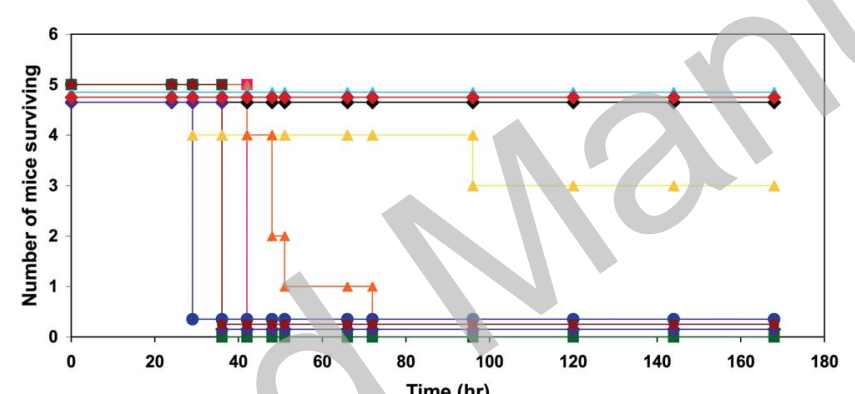

- - ExoS(E379A/E381A)

- Exos(L423A)

- Exos(L426AD427ALL428A) - ExoS(H418A)

足

Licenced copy. Copying is not permitted, except with prior permission and as allowed by law.

() 2010 The Authors Journal compilation @ 2010 Portland Press Limited 